\title{
Epidemiology and clinico-pathological characteristics of current goat pox outbreak in North Vietnam
}

\begin{abstract}
Background: In view of the current swine fever outbreak and the government aspiration to increase the goat population, a need arises to control and prevent outbreaks of goat pox. Despite North Vietnam facing sporadic cases of goat pox, this most recent outbreak had the highest recorded morbidity, mortality and case fatality rate. Thus, owing to the likelihood of a widespread recurrence of goat pox infection, an analysis of that outbreak was done based on selected signalment, management and disease pattern (signs and pathology) parameters. This includes examination of animals, inspection of facilities, tissue sampling and analysis for confirmation of goatpox along with questionaires. Results: It was found that the susceptible age group were between 3 and 6 months old kids while higher infection rate occurred in those under the free-range rearing system. The clinical signs of pyrexia, anorexia, nasal discharge and lesions of pocks were not restricted to the skin but have extended into the lung and intestine. The pathogen had been confirmed in positive cases via PCR as goat pox with prevalence of $79.69 \%$. Conclusions: The epidemiology of the current goat pox outbreak in North Vietnam denotes a significant prevalence which may affect the industry. This signals the importance of identifying the salient clinical signs and post mortem lesions of goat pox at the field level in order to achieve an effective control of the disease.
\end{abstract}

Keyword: North Vietnam; Goat pox; Epidemiology; Pathology; Industry 